\title{
The Science Policy of the Joint Institute for Nuclear Research
}

\author{
A. Sissakian
}

\section{Dear Colleagues,}

It is great honor for me to participate in this conference, devoted to the 70th anniversary of the birth of the prominent Czech scientist Professor Jiří Niederle.

Following the title of my talk, I'll give you an introductory presentation about JINR's strategic development plans, starting with a very brief historical background.

The Joint Institute for Nuclear Research (JINR) is located in the Moscow Region, in Dubna, on the bank of the Volga river $(120 \mathrm{~km}$ to the north of Moscow). The Institute was established in March 1956 through a convention signed by the Plenipotentiaries of the governments of the JINR Member States. The Institute was created in order to unify the intellectual and material potential of the Member States in order to study the fundamental properties of matter.

Until the early 1990s, Dubna was a centre that unified the efforts of leading research groups in the nuclear sciences from the socialist countries and the Soviet Union.

A new development stage in the history of the Institute has started in 1992. 18 independent states, including 9 republics of the former USSR, became its Member States. In addition, agreements at governmental level were signed for cooperation with 6 countries - Germany, Hungary, Italy, the Republic of South Africa, Serbia and, recently, with Egypt.

It is well-known that the future is based on the past, and on traditions. JINR has a huge foundation based on three "pillars":

- great experience in nuclear physics research and the world-wide recognized traditions of its scientific schools

- a large and unique park of basic facilities for fundamental and applied research

- the status of an international intergovernmental organization

For over 50 years of JINR's existence, first-class theoretical and experimental research programmes implemented at JINR have led to a significant scientific output in fundamental nuclear science. More than 40 discoveries in nuclear physics, particle physics and condensed matter physics have been made in the JINR laboratories.

In recognition of the achievements of JINR's staff of researchers, in 1997 the International Committee for Pure and Applied Chemistry awarded the name "Dubnium" to element 105 of the Periodic Table of Elements. Among the latest bright results, I would like to mention the pioneering investigations of the chemical properties of the superheavy elements.

The main governing body of the Institute is the Committee of Plenipotentiaries of the Member States' Governments. The scientific policy is established and co-ordinated by the Scientific Council, whose members are prominent and well-known scientists from the Member States as well as from CERN, Germany, Greece, France, Italy, China, India and other countries. Three Programme Advisory Committees for Particle Physics, Nuclear Physics and Condensed Matter Physics hold their meetings twice a year.

There are seven Laboratories at JINR, each being comparable to a large research institution in terms of the scope of scientific activities. We also have a University Centre, as well as several functional subdivisions and workshops.

\section{Present Scientific Policy}

The triad: fundamental research, innovation developments and the educational programme - forms the strategic policy line of the Institute's development.

I would like to note that, along with the current 7-year programme of the Institute's development for the years 2003-2009 (and now we are developing the next 7-year programme for the years $2010-2016),{ }^{1}$ we have elaborated the programme of JINR's strategic development (the so-called Road Map) for the next 10-12 years. This programme takes into account both the world tendencies in scientific developments and the interests of our Member States.

Fundamental studies will remain as the general direction of JINR's development. At the same time, special attention will be given to innovation activities, in particular to radiation medicine, nanotechnologies and others. The innovative projects will be developed in close cooperation with the Public-Private-Partnership in the framework of the Special Economic Zone in Dubna. The role of the educational programme will be further enhanced.

The Road Map has determined three major research directions at JINR: high energy physics, nuclear

\footnotetext{
${ }^{1}$ Approved in September 2009 by the JINR Scientific Council, and in November 2009 by the JINR Committee of Plenipotentiaries of the governments of the Member States
} 
physics and condensed matter physics. As I have already mentioned, the activities in these directions are implemented at our seven laboratories.

Such disciplines as theory, network and computing, as well as physics instrumentation and methods, training of the young generation are very important supporting activities for our basic research directions.

One of the key points of the Road Map is that JINR should develop its role as a world leader in certain research domains. Therefore, it is necessary to formulate the priority trends in the development of the Institute.

To achieve this ambitious goal, we understand clearly that the whole experimental infrastructure of the Institute, and first of all our basic facilities, must be completely upgraded. Such a programme will allow us to become a competitive scientific laboratory, attractive, first of all, for the Member States, for the countries with which we have tight and long-standing cooperation activities, e.g. Czechia, Hungary, Germany, France, Italy and other partners.

While updating the Road Map we clearly see that the research niches for JINR, offered by our home facilities, are the following: Heavy Ion Physics at high energies and at low energies, and condensed matter physics using nuclear-physics methods, including radiobiology studies.

Now briefly about our large-scale facilities and our ambitious research projects.

In Heavy Ion Physics at High Energies, the new flagship of the Institute and the highest priority project is the NICA/MPD (Nuclotron-Based Ion Collider fAcility and MultiPurpose Detector) with energy of $4-11 \mathrm{GeV}$ in the nucleon-nucleon center-of-mass. This project is aimed at the study of hot and dense strongly interacting QCD matter and the search for a possible manifestation of mixed phase formation in heavy ion collisions, as well as studies on spin physics.

This energy range is of great interest and importance for understanding the evolution of the Early Universe in the first milliseconds after the Big Bang, and the formation of neutron stars as well. To achieve these physics goals on NICA, we have to accomplish several large technical tasks. First of all, upgrading of the Nuclotron itself, achieving its project parameters by 2010 . This will be the first step towards the NICA collider facility. The next two steps deal with the design and construction of the heavy ion collider itself and the MultiPurpose Detector. The key advantage of the NICA project is the sufficiently high luminosity in the energy range of $4-11 \mathrm{GeV}$ in the nucleonnucleon center-of-mass $\left(\sim 10^{27} \mathrm{~cm}^{-2} \mathrm{sec}^{-1}\right.$ at $9 \mathrm{GeV})$.

The NICA complex will comprise several accelerators and beam transfer channels. The design of the collider suggests two intersection points, where the detectors are to be located. One of them, the MultiPurpose Detector (MPD), is dedicated to Mixed Phase problem studies. One of the options for another detector is a Spin Physics Detector (SPD).

Table 1: NICA general parameters

\begin{tabular}{|l|c|}
\hline Ring circumference & $251.52 \mathrm{~m}$ \\
\hline $\mathrm{B} \rho \max$ & $45.0 \mathrm{~T} \cdot \mathrm{m}$ \\
\hline $\begin{array}{l}\text { Ion kinetic energy } \\
(\text { Au79+) }\end{array}$ & $1.0 \div 4.56 \mathrm{GeV} / \mathrm{u}$ \\
\hline Dipole field (max) & $4.0 \mathrm{~T}$ \\
\hline $\begin{array}{l}\text { Free space at } \\
\text { interaction point } \\
\text { (for detector) }\end{array}$ & 0 \\
\hline $\begin{array}{l}\text { Beam crossing angle } \\
\text { at interaction point }\end{array}$ & $10^{-11} \mathrm{Torr}$ \\
\hline Vacuum & $0.75 \div 11 \cdot 10^{26} \mathrm{~cm}^{-2} \cdot \mathrm{s}^{-1}$ \\
\hline $\begin{array}{l}\text { Luminosity per one } \\
\text { interaction point }\end{array}$ & \\
\hline
\end{tabular}

The central goal of the heavy ion experimental studies is to explore the phase diagram of nuclear matter. It is expected that it has at least two different phases: hadronic and quark-gluon phases, and they are separated by a first order phase transition at high baryon densities.

In the plane of temperature and baryon-density the first order phase transition results in a finite width band of the coexistence region of these two phases, the so-called mixed phase. We attracted the attention to this remarkable fact in the middle of 2005 .

Exploration of the mixed phase region is one of the goals of the future NICA/MPD and FAIR/CBM (Compressed Baryonic Matter) experiments, together with the low energy scan program at RHIC and the NA61 experiment at SPS. This region has not been adequately explored up to now.

Among our external research activities I would like to mention two experiments with JINR's most active participation.

The first of these is the CERN NA48/2 experiment.Very large statistics of high performance data of charged kaon decays has been accumulated by this experiment at the CERN SPS. Two major parameters $-\boldsymbol{a}_{0}$ and $\boldsymbol{a}_{2}$ - of scattering lengths have been extracted with an unprecedented experimental precision of a few percent, allowing accurate tests of Chiral Perturbation theory predictions. This result was highlighted at CERN as an experimental achievement in 2008.

The other experiment deals with our activities at Fermilab. JINR physicists, together with their American colleagues, performed a physical analysis in 2008 of the D0 experiment at the Tevatron, which led to the first observation of the $\Omega_{b}$ baryon. This discovery was ranked among the ten most significant achievements in physics in 2008 by the Amer- 
ican Physics Society, and we are again proud that JINR made a significant contribution to this analysis.

JINR successfully completed all its obligations to the three LHC projects: ATLAS, ALICE and CMS. The total contribution of JINR to these projects, including LHC Damper, amounted to approximately 25 million Swiss francs. Concerning JINR-CERN collaboration, I'd like to note that in accordance with our recent negotiations with the new CERN DirectorGeneral R. Heuer, our collaboration will be of high priority for both centres. Now we are preparing a renewed version of the general Partnership Agreement to be signed at the beginning of $2010^{2}$.

As for nuclear physics (low and intermediate energy heavy ion physics), I'd like to mention that we are proud that in the last decade JINR has become one of the leading scientific centres in the world in the synthesis of superheavy elements and physical and chemical investigations of these nuclei. Other priority topics in this domain are the structure and properties of neutron-rich light exotic nuclei, accelerator technology and broad applied research, including nanotechnology.

For the past 7-year period in a number of experiments performed with the use of the intense beam of ${ }^{48} \mathrm{Ca}$ and actinide targets, 5 new elements and 34 new superheavy isotopes have been synthesized. These experiments have been carried out in wide collaboration with scientific centers in Russia and the USA. Chemical properties of elements $112-115$ were established for the first time. In particular, it was shown that elements 112 and 114 are more volatile than their lighter homologues, due to relativistic effects in the electronic structure. So, a new branch of science really is presently developing at JINR - Relativistic Chemistry of Superheavy Elements.

Neutrino physics is a traditional research topic for our Institute. One of the most fundamental studies in neutrino physics is the search for neutrinoless double beta decay. Very impressive results have been obtained by the NEMO-3 Collaboration. At present the limit for neutrino mass established by this experiment is of the order of $1 \mathrm{eV}$. It is planned in 2009-2010 to go down by a factor of two in this limit. In 2011 the installation of the Super NEMO detector will start. This new detector will provide sensitivity for the search for neutrinoless double beta decay below $0.1 \mathrm{eV}$ in the neutrino mass.

As for condensed matter physics', I'd like to mention that JINR's basic facility here is the IBR-2 fast pulsing reactor. It is included in the European 20-year strategic research programme with neutrons. The reactor is now under an upgrade programme. In 2010, the practically new IBR-2M reactor will start up its operation.

The main priorities in condensed matter physics in the next 7-year period deal with reliable operation at design parameters of the new IBR-2M reactor, the establishment of a complex of modern neutron spectrometers, realization of a full-scale cryogenic complex, as well as wide-ranging innovations including nanoscience. Special attention will be given to further development of the spectrometer complex of the IBR-2M reactor. The first priority projects include the construction of the new DN-6 spectrometer for studies of microsamples, the GRAINS multifunctional reflectometer, and modernization of the spectrometer complex for geophysical research. These facilities will significantly expand the area of world-level research at the IBR-2M reactor. We expect a broad international user policy on this machine, as in the past, or even broader.

The radiobiology studies at JINR are aimed at obtaining fundamental results which will be principally important to general and cosmic radiobiology. Starting from 2009, our Laboratory of Radiobiology has conducted its research programme under the scientific and methodological supervision of the Section of Biological Sciences of the Russian Academy of Sciences.

It is important to take into our consideration that in this field of our activities we have to employ the huge scientific potential of the Joint Institute for the progress of this trend at the interface of biology, medicine and physics. Obviously, we have to expect innovation breakthroughs exactly in this area during the next evolution cycle of civilization.

The educational programme plays an important role in the JINR's activities. In 1991 we established the JINR University Centre and in 1994, together with the Russian Academy of Natural Sciences, the Dubna International University. This provides good opportunities for young scientists to get their postgraduate training, obtain $\mathrm{PhD}$ degrees and receive advanced professional training using JINR's experimental facilities.

The Dubna International Advanced School on Theoretical Physics came into operation in 2003.

A few words about the innovation activities in our Institute. In 2005 the Russian Government adopted a resolution on the establishment of six Special Economic Zones (SEZ) in the territory of the Russian Federation. The status of a special economic zone of technological and innovative type was granted to four towns: Zelenograd, Dubna, St. Petersburg and Tomsk.

The main specializations of the Dubna SEZ are related to nuclear physics and IT technologies, including radiation medicine, safety systems, nanotechnologies and IT technologies.

On 18 April 2008, President of the Russian Federation Dmitry Medvedev chaired a session of the Presidium of the State Council in Dubna which discussed the issue "Development of the National Innovation System in the Russian Federation".

\footnotetext{
${ }^{2}$ Agreement has been signed on 28 February 2010.
} 
During his visit, President D. Medvedev highly appreciated the results of JINR's basic research and underlined the role of science in the innovation process.

The President noted the importance of the future realization of two large-scale projects, proposed by JINR: the establishment in Dubna of a Centre for Radiation Medicine and of an International Innovation Centre for Nanotechnology.

I would like to note that over the decades JINR has played the role of a cluster centre for its Member States, giving them an efficient way to be integrated into the European scientific society (for example CERN, INFN and others).

We hope that the International Innovative Centre for Nanotechnology, which we are now creating, will also serve for the integration of European innovation activities.

In particular, you know that in 2008 the European Institute for Innovation and Technology was established by the European Union, with its General Headquarters in Budapest, Hungary. We intend to include our Innovation Centre in its Knowledge and Innovation Communities (KIC) as a first step in the integration process.

I believe that our cooperation with the Member States will further serve our mutual interests and, undoubtedly, will strengthen the scientific ties in both basic and applied research and will integrate our joint research into European science.

Concluding, let me say a few words about cooperation activities between JINR and Czechia. The Czech Republic has always been one of the most active Institute Member States. It should be mentioned that Czech scientists have served as JINR Vice-Directors, Deputy Directors of the JINR laboratories, heads of departments and groups: V. Votruba, V. Petrzilka, C. Simane, I. Zvara and many others.

Taking the opportunity, I would like to cordially congratulate Prof. Cestmir Simane on his forthcoming 90th birthday, and wish him good health and happiness.
Today JINR conducts cooperation with 9 Czech scientific centres, 3 Universities and the company Vacuum-PRAGUE on 34 scientific topics in all JINR scientific trends.

JINR's main partners in the Czech Republic are: the Nuclear Physics Institute in Rez, Charles University, the Czech Technical University, the Institute of Physics in Prague, the company Vacuum Prague, and many others.

A ceremonial meeting dedicated to the 50th anniversary of JINR was held on 24 January in Prague, in the building of Charles University, one of the oldest universities in Europe.

Among the honorary guests present at the meeting were Prime Minister of the Czech Republic J. Paroubek, Plenipotentiary of the Russian Federation to Czechia A. Fedotov, as well as representatives of Czech scientific, governmental and social organizations.

Prime Minister of Czechia J. Paroubek congratulated the audience on the 50th anniversary of JINR and noted the great role of the Dubna international physics centre in founding and developing nuclear physics in all countries - JINR Member States, including Czechoslovakia and Czechia.

Professor J. Niederle has contributed greatly to our cooperation. An outstanding theoretician, he has for many years been promoting the development of our collaboration in theoretical physics. As Scientific Secretary of the Presidium of the Czech Academy of Sciences, he contributed much to the solution of strategic issues in our joint activities.

Please, accept once more my heartiest congratulations on the occasion of Professor Jiři Niederle's anniversary.

Alexey Sissakian

Academician, Director of JINR 